\title{
Evaluation of the surface affinity of water in three biochars using fast field cycling NMR relaxometry
}

\author{
Salvatore Bubici, ${ }^{a, e}$ Jean-Pierre Korb, ${ }^{b}$ Jiří Kučerik $^{c}$ and Pellegrino Conte ${ }^{d_{*}}$
}

\begin{abstract}
Many soil functions depend on the interaction of water with soil. The affinity of water for soils can be altered by applying soil amendments like stone meal, manure, or biochar (a carbonaceous material obtained by pyrolysis of biomasses). In fact, the addition of hydrophobic biochar to soil may increase soil repellency, reduce water-adsorbing capacity, inhibit microbial activity, alter soil filter, buffer, storage, and transformation functions. For this reason, it is of paramount importance to monitor water affinity for biochar surface (also referred to as 'wettability') in order to better address its applications in soil systems. In this study, we propose the use of fast field cycling NMR relaxometry technique with the application of a new mathematical model for data interpretation, as a valid alternative to the traditional contact angle (CA) measurements for biochar wettability evaluation. Either NMR or CA results revealed the same wettability trend for the biochars studied here. The advantage of NMR relaxometry over CA measurements lies in the possibility to obtain at the microscopic level a variety of different information in only one shot. In fact, while CA provides only wettability evaluation, NMR relaxometry also allows achievement of the mechanisms for water molecular dynamics on biochar surface, thereby leading to the possibility to understand better, in future research, the role of biochar in increasing soil quality and plant nutrition. Copyright $\odot 2016$ John Wiley \& Sons, Ltd.
\end{abstract}

Keywords: NMR; 1H; relaxometry; fast field cycling; biochar; water affinity; wettability

\section{Introduction}

For thousands of years, in all known civilizations, biochar (a carbonaceous material obtained by deliberate pyrolysis of biomasses) has been used as a basic material for applications such as cooking, space heating, forging of metal tools, soil amendment, animal bedding, medicine, and feed additive. ${ }^{[1]}$ In the last decade, this material has become the major research topic for many scientific groups because of its potential impact on slowing global warming and on its capacity to restore degraded soils. ${ }^{[2-8]}$ In fact, photosynthesis produces carbonaceous plant materials, which return back to $\mathrm{CO}_{2}$ when plant materials are allowed to decompose. However, if the harvested plant residues are pyrolyzed, up to $60 \%$ of the original plant carbon can be transformed into biochar. In soils, biochar mineralization to carbon dioxide is extremely slow, thereby providing a pathway for long-term subtraction of $\mathrm{CO}_{2}$ from the global carbon cycle and reducing the concentration of $\mathrm{CO}_{2}$ in the atmosphere. ${ }^{[5]}$ Moreover, biochar application to soils appears also very effective in improving soil quality and crop production through mechanisms, which are still poorly understood. ${ }^{[5,9,10]}$

It has been shown that porosity plays the major role in retaining different amounts of water in biochar systems. ${ }^{[7,8]}$ In particular, water in biochar undergoes 2D surface diffusion as it is constrained in micro-pores, while, when pore size enlarges, water-surface interactions become weaker, and 3D exchange with the bulk liquid occurs. ${ }^{[6,8]}$ Moreover, water retention in biochar amended soils appears mainly affected by intra-aggregate porosity (i.e. pores spaces between particles within the fabric units, textural pores, and micropores) when low amounts of biochar are added to soils, whereas swelling processes driven by inter-aggregate porosity (i.e. pore spaces between fabric units, structural pores, and macropores) become important when biochar concentration in soils increases. ${ }^{[7]}$

The physical-chemical mechanisms of water retention in biochar appear to be affected by formation of weak interactions mediated either by the electron deficiencies of the metals in the biochar inorganic constituents or by the $\pi$-clouds of the aromatic carbon system. In fact, electron-deficient metals may form charge-transfer bindings with the electron-rich oxygen present in water, whereas unconventional $\mathrm{H}$-bonds occur via electron donation from the $\pi$-clouds of the poly-condensed aromatic systems towards the electron-deficient hydrogens in water. ${ }^{[11]}$

It is worth noting that the strength of the aforementioned interactions may change according to the surface functional groups involved in the interactions. In fact, as strong electron donors

* Correspondence to: Pellegrino Conte, Dipartimento di Scienze Agrarie e Forestali, Università degli Studi di Palermo, v.le delle Scienze edificio 4, 90128 Palermo, Italy. E-mail: pellegrino.conte@unipa.it

a Stelar srl, 27035, Mede, PV, Italy

b Physique de la Matière Condensée, Ecole Polytechnique-CNRS, 91128, Palaiseau, France

c Institute for Environmental Sciences, Department of Soil and Environmental Chemistry, University of Koblenz-Landau, Landau 76829, Germany

d Dipartimento di Scienze Agrarie e Forestali, Università degli Studi di Palermo, 90128, Palermo, Italy

e JEOL (ITALIA) S.p.A., Palazzo Pacinotti-Milano 3 City, Via Ludovico il Moro 6/A, 20080, Basiglio, MI, Italy 
(e.g. oxygen and nitrogen atoms in oxygenated and nitrogenated functions, respectively) are still present, when low temperatures are used for biochar production, ${ }^{[12]}$ affinity of water for biochar surface enhances (i.e. stronger interactions occur). Conversely, when biochar production temperature is increased, water affinity for biochar surface decreases (i.e. weaker interactions occur).

In general, water affinity for biochar is also referred to as biochar wettability. ${ }^{[13-15]}$ Wettability can be considered as a qualitative indication of the strength of the water-biochar interface interactions. The stronger the liquid-solid interface interactions, the larger the wettability is. Conversely, as the aforementioned interactions become weaker, wettability decreases. As a general remark, wettability represents one of the most important parameters determining the role of biochar in soils. As a matter of fact, the presence of inappropriate biochar type and application amount (due either to deliberate application for soil quality enhancement or to uncontrolled forest fires) can cause soil repellency, thereby affecting soil's capacity to absorb water, inhibiting microbial activity, altering filter, buffer, storage, and transformation functions of the soils. ${ }^{[16-18]}$

Up to now, the only direct macroscopic way to measure wettability of biochar has been through the evaluation of contact angle (CA). ${ }^{[13-15]}$ The latter is the angle between the intersection of the liquid-solid and the liquid-vapor interfaces. Geometrically, it is acquired by applying a tangent line from the contact point along the liquid-vapor interface in the droplet profile ${ }^{[19]}$ (Fig. 1). The technique is based on the surface tension of the liquid. In fact, surficial molecules in a liquid do not have neighboring molecules in all directions to provide a balanced net force. Instead, they are pulled inward by the neighboring molecules, thereby creating an internal pressure. As a result, the liquid contracts its surface area to maintain the lowest surface free energy. ${ }^{[19]}$ The more affine to the liquid the solid surface is, the more spread is the liquid on the solid surface (Fig. 1A). Conversely, when affinity between the two phases decreases, the liquid beads the solid (Fig. 1B). As a consequence, high wettability is achieved when small CAs (i.e. $<90^{\circ}$ ) are measured, whereas low wettability is retrieved when CAs are large (i.e. $>90^{\circ}$ ) (Fig. 1).

A series of nuclear magnetic resonance (NMR) techniques have been applied to characterize the nature and chemical composition of biochars. ${ }^{[20-22]}$ While the traditional NMR spectroscopy reveals that the molecular composition of biochar depends upon biomass feedstock, production procedure, and temperature, ${ }^{[6,12,21]}$ a more
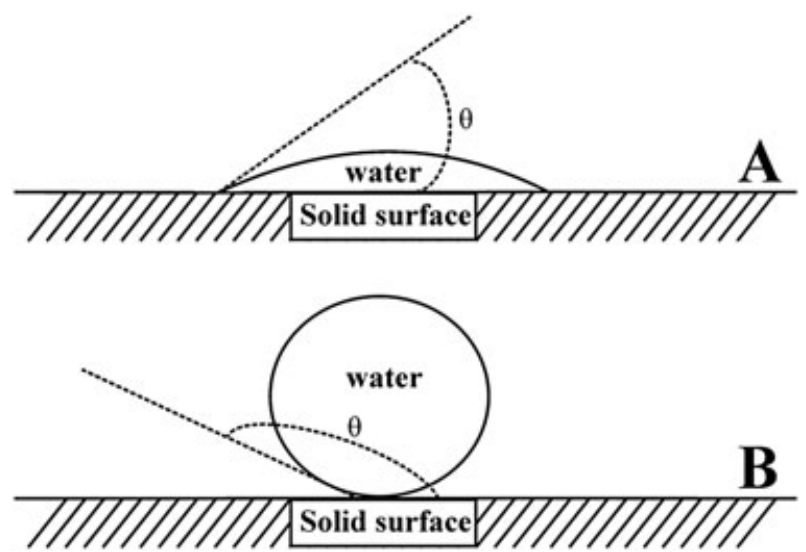

Figure 1. A. Interaction between water and a hydrophilic solid surface. B. Interaction between water and a hydrophobic solid surface. $\theta$ is the contact angle. recent NMR technique, referred to as fast field cycling (FFC) relaxometry, has been applied in order to recognize at microscopic level molecular dynamics of water and nutrients on biochar surfaces. ${ }^{[22]}$

Fast field cycling NMR relaxometry deals with the variation of the spin-lattice relaxation times $\left(T_{1}\right)$ in a complex system, as the strength of the constant applied magnetic field is changed. In particular, $T_{1}$ values are monitored as a function of the fluctuations of the local magnetic fields generated by nuclear dipoles, unpaired electrons, electric charges interacting with nuclear quadrupole moments for $>1 / 2$ spin nuclei, anisotropy of the chemical shielding tensor, fluctuating scalar coupling interactions, and molecular rotations. As a general remark, FFC NMR relaxometry allows achievement of motion frequencies in the range $\sim 10^{4}$ to $\sim 40 \times 10^{6} \mathrm{~Hz}$, thereby consenting evaluation of molecular dynamics, which are not accounted for by the traditional high field NMR spectroscopy. ${ }^{[22]}$

The aim of the present paper is to suggest the use of FFC NMR relaxometry as a valid alternative to CA measurements for the evaluation at the microscopic level of biochar wettability. In particular, a model designed for understanding water mobility in inorganic porous systems ${ }^{[23]}$ has been accounted for the interpretation of FFC NMR biochar data. Conclusions resulted more suitable than those achieved by the free model analysis ${ }^{[24]}$ applied in a previous investigation, ${ }^{[25]}$ thereby suggesting not only that the new FFC NMR relaxometry model can be successfully applied to monitor wettability of organic complex systems such as biochar, but also that the wettability mechanisms are invariant from a nano-scale up to a macro-scale dimension.

\section{Materials and methods}

\section{The biochar samples}

Biochars were obtained by the gasification procedure of three different feedstock: conifer wood chips, poplar wood chips, and grape press residues (grapevine marc). All the feedstock, as well as all biochar characteristics used to achieve the data reported in Table 1, have been already described in De Pasquale et al. ${ }^{[25]}$ For this reason, readers are asked to refer to the aforementioned paper for details on the techniques applied for biochar characterization. In the present paper, we describe only details on CA measurements not reported previously, and the FFC NMR relaxometry acquisition and elaboration procedures, which were modified as compared with the previous study. ${ }^{[25]}$

Table 1. Surface area $\left(S_{p}\right)$, number of paramagnetic ferric ions per gram of biochar $\left(\eta_{s}\right)$, number of paramagnetic ferric ions per biochar surface unit $\left(\sigma_{s}\right)$ defined from $\eta_{s}$ and $\rho$ assuming a uniform distribution of $\mathrm{Fe}^{3+}$ ions, biochar bulk density $(\rho)$, contact angle $(\theta) . \eta_{s,}$ and $\sigma_{s}$, have been obtained by the chemical analyses reported in De Pasquale et al. ${ }^{[25]}$

\begin{tabular}{lccc|} 
Parameters & $\begin{array}{c}\text { Conifer } \\
\text { biochar }\end{array}$ & $\begin{array}{c}\text { Poplar } \\
\text { biochar }\end{array}$ & $\begin{array}{c}\text { Grapevine } \\
\text { marc biochar }\end{array}$ \\
\hline$\theta\left(^{\circ}\right)$ & $128 \pm 4$ & $114 \pm 1$ & $114 \pm 2$ \\
$\mathrm{~S}_{p}\left(\mathrm{~m}^{2} \cdot \mathrm{g}^{-1}\right)$ & $66 \pm 5$ & $98 \pm 6$ & $42 \pm 4$ \\
$\eta_{s}\left(\mathrm{Number} \mathrm{Fe}^{3+} \cdot \mathrm{g}^{-1}\right)$ & $1.94 \cdot 10^{19}$ & $6.15 \cdot 10^{18}$ & $8.09 \cdot 10^{19}$ \\
$\sigma_{s}\left(\mathrm{Number} \mathrm{Fe}^{3+} \cdot \mathrm{cm}^{-2}\right)$ & $2.43 \cdot 10^{12}$ & $1.88 \cdot 10^{12}$ & $1.64 \cdot 10^{12}$ \\
$\rho\left(\mathrm{g} \cdot \mathrm{cm}^{-3}\right)$ & 0.196 & 0.421 & 0.341 \\
\hline
\end{tabular}




\section{Contact angle measurements (Wilhelmy plates method)}

The CA was determined by using the Wilhelmy plate method ${ }^{[26]}$ by applying the Dynamic Contact Angle Meter and Tensiometer Serie 21 (DataPhysics). Prior to analyses, all the grounded biochar samples were dried at $30^{\circ} \mathrm{C}$ for $24 \mathrm{~h}$. Afterwards, a double-faced adhesive tape was fixed on the upper third on both sides of a microscope slide, and the samples were spread all over the adhesive tape with a spatula. The excess of non-glued samples was carefully removed, and the slide was dived into a container with water. The force needed to pull out the slide from the water was determined by weighing the slide during pulling. The CA ( $\theta$ in Fig. 1) was calculated according to Eqn (1):

$$
\theta=\arccos \left(\frac{\Delta F}{L \sigma}\right)
$$

$\Delta F$ is the aforementioned force determined from weight variation, $L$ is the wetted length of the microscope slide, and $\sigma$ is the water surface tension, which results $72 \times 10^{-3} \mathrm{Nm}^{-1}$ at $25^{\circ} \mathrm{C}^{[27]} \mathrm{CAs}$ for the three biochar samples used in the present study are reported in Table 1 . They were obtained by averaging triplicate results. As a general remark, the smaller the $C A$, the more hydrophilic is the surface of the porous medium. Conversely, the increase in CA reflects higher hydrophobicity of the investigated surfaces.

\section{FFC NMR relaxometry experiments}

One gram of each biochar sample was suspended in $3 \mathrm{ml}$ of deionized water and subjected to FFC NMR relaxometry investigation. In particular, all the ${ }^{1} \mathrm{H}$ NMR dispersion profiles (i.e. relaxation rates $R_{1}$ or $1 / T_{1}$ vs proton Larmor frequencies) were acquired on a Stelar Spinmaster FFC2000 Relaxometer (Stelar s.r.l., Mede, PV - Italy) at $25^{\circ} \mathrm{C}$. The details of the basic FFC NMR experiments on environmental samples have been reported by Conte and Alonzo. ${ }^{[22]}$ Briefly, the proton spins were polarized at a polarization field $\left(\mathrm{B}_{\mathrm{POL}}\right)$ corresponding to a proton Larmor frequency $\left(\omega_{L}\right)$ of $24 \mathrm{MHz}$ for a period of polarization ( $T_{\mathrm{POL}}$ ) corresponding to about five times the $T_{1}$ estimated at this frequency. After each $B_{P O L}$ application, the magnetic field strength (indicated as $B_{R L X}$ ) was systematically changed in the proton Larmor frequency comprised in the range $0.01-40.0 \mathrm{MHz}$. The period $\tau$, during which $\mathrm{B}_{\mathrm{RLX}}$ was applied, has been varied on 64 logarithmic spaced time sets, each of them ranged between $1 \mathrm{~ms}$ up to $10 \mathrm{~s}$ in order to sample all the possible relaxing components analyzable in the decay/recovery curves. Free induction decays were recorded following a single ${ }^{1} \mathrm{H} 90^{\circ}$ pulse applied at an acquisition field $\left(\mathrm{B}_{\mathrm{ACQ}}\right)$ corresponding to the proton Larmor frequency of $35 \mathrm{MHz}$. Field-switching time was $3 \mathrm{~ms}$, while spectrometer dead time was $15 \mu \mathrm{s}$. For all experiments, a recycle delay of $12 \mathrm{~s}$ was used. A non-polarized FFC sequence was applied when the relaxation magnetic fields were in the range of the proton Larmor frequencies comprised between 40.0 and $8.0 \mathrm{MHz}$. A prepolarized FFC sequence was applied in the proton Larmor frequencies $\mathrm{B}_{\mathrm{RLX}}$ range of $8.0-0.01 \mathrm{MHz}$.

All the decay/recovery curves acquired as aforementioned, were transformed with the inverse Laplace function by applying the Uniform PENalty regularization (UPEN) algorithm. ${ }^{[22]} \mathrm{A}$ bimodal distribution of water molecules experiencing different environments was identified in each biochar/water mixture. The narrow distribution of water molecules centered about $T_{1} \sim 2.5 \mathrm{~s}$ in the whole range of $B_{R L X}$ field strengths were identified as bulk water. This latter value is due to the oxygen dissolved in bulk water. Conversely, the molecules relaxing at the shortest $T_{1}$ values and providing a NMR dispersion (NMRD) profile were associated with water directly in contact (either mobile or immobile) with the biochar surface. ${ }^{[22]}$ In the discussion later, only this second type of water molecules was accounted for.

\section{The mathematical model used for the elaboration of the NMRD profiles}

As biochar samples are suspended in water, two different mechanisms for water mobility occur. ${ }^{[6,8,11]}$ On the one hand, water molecules predominantly undergo $2 \mathrm{D}$ diffusion on biochar surface when the main pores are storage-like (i.e. $0.5-50 \mu \mathrm{m}$ ) and residual-like (i.e. $<0.5 \mu \mathrm{m}$ ) ones. On the other hand, 3D exchanges between unbound and bound water prevalently occur when transmission-like pores (i.e. $\geq 50 \mu \mathrm{m}$ ) are present. The two mechanisms are not mutually exclusive to each other because of the biochar multi-micron size porosity. ${ }^{[8]}$ In fact, all biochars contain pores whose dimensions range from few up to tens of microns, regardless of their texture size. ${ }^{[8]}$

The distribution of water motional frequencies depends upon the homogeneity of the surface of the porous medium. In fact, water confined in small sized pores is more tightly constrained than that freely moving in larger spaces. ${ }^{[22]}$ The distributions of magnetic fields generated by the motional fluctuations are responsible for the dispersion of the longitudinal (or spin-lattice) relaxation times $\left(T_{1}\right)$ occurring when each frequency in distributions of magnetic fields matches the Larmor frequencies $\left(\omega_{L}\right)$ of the observed nuclei (i.e. ${ }^{1} \mathrm{H}$ ). Water near the surface can also interact with surficial paramagnetic ions. The resultant modulation of the local dipolar magnetic field generated by paramagnetism additionally contributes to spin-lattice relaxation. ${ }^{[23]}$

Previously, Korb et al. ${ }^{[23]}$ introduced a model to explain the dynamics of liquids on the surface of porous systems in the presence of rare paramagnetic impurities. In particular, their model describes the variation of the longitudinal relaxation rate according to Eqn (2):

$R_{1}\left(\omega_{l},, \omega_{S}\right)=R_{0}+K \times \tau_{m}\left[3 \ln \left(\frac{1+\omega_{l}^{2} \tau_{m}^{2}}{\left(\tau_{m} / \tau_{s}\right)^{2}+\omega_{l}^{2} \tau_{m}^{2}}\right)+7 \ln \left(\frac{1+\omega_{s}^{2} \tau_{m}^{2}}{\left(\tau_{m} / \tau_{s}\right)^{2}+\omega_{s}^{2} \tau_{m}^{2}}\right)\right]$

Here, $\omega_{l}$ is the proton Larmor frequency, while $\omega_{S}=659 \omega_{\text {, }}$ is the Larmor frequency of the unpaired electron in the paramagnetic species. $R_{0}$ represents the sum of the water bulk relaxation time of the order of $2.5 \mathrm{~s}$ and the frequency independent water-bound contribution, which is affected by the amount of water binding sites. The term in squared brackets in Eqn (2) represents the surface contribution corresponding to the surface water diffusion in proximity of the paramagnetic source of relaxation ( $\mathrm{Fe}^{3+}$ or others). In Eqn (2), $K$ is given by the relation

$$
K=\frac{\pi}{30 \delta_{\text {water }}^{3}} \sigma_{S} \rho_{\text {water }} S_{P, N M R}\left(\gamma_{1} \gamma_{S} \hbar\right)^{2} S_{S}\left(S_{S}+1\right) .
$$

In relation (3), $\delta_{\text {water }}=0.3 \mathrm{~nm}$ is the average water molecular size, $\rho_{\text {water }}=1 \mathrm{~g} \mathrm{~cm}^{-3}$ is the water density, $\sigma_{s}$ is the density of the paramagnetic ions at the pore surface (i.e. sources of relaxation expressed in number of paramagnetic spins per $\mathrm{cm}^{2}$ ) probed by electron spin resonance, $S_{p, N M R}$ (expressed in $\mathrm{m}^{2} \mathrm{~g}^{-1}$ ) is the NMR specific surface area given by $S_{p} F\left(S_{p}\right.$ is the surface area achieved by the Brubauer-Emmet-Teller, analysis already described in De Pasquale et al., ${ }^{[25]}$ while $F<1$ is the solid-to-liquid ratio), $\gamma_{l}$ and 
$\gamma_{S}=659 \gamma_{I}$ are the proton and electron (for the most abundant paramagnetic species) magnetogyric ratios, respectively. ${ }^{[23]}$ Finally, $S_{S}=5 / 2$ for $\mathrm{Fe}^{3+}$ is the spin quantum number of the paramagnetic species. ${ }^{[23]} \tau_{m}$ is the surface diffusion correlation time, while $\tau_{s}$ is indicated as surface residence time. ${ }^{[23]}$ The former represents the 'hopping' time of water among surficial binding sites, while the latter $\left(\tau_{s}\right)$ is the time of residence of water on the surface of the porous medium (see discussion later for further details).

In the present study, Eqn (2) has been applied to fit the NMRD profiles of the logarithmic average of the fastest relaxing components revealed as aforementioned. The parameters reported in relation (3), and used to fit the data, are reported in Table 1. They were calculated according to the analyses reported in De Pasquale et al. ${ }^{[25]}$

\section{Results and discussion}

\section{CA measurements}

Water molecules form an infinite hydrogen-bonded network with localized and well-structured clustering. ${ }^{[28]}$ As they approach the surface of a solid system, two different limiting mechanisms may occur. In particular, in the case of hydrophilic surfaces, the adhesion forces, due to formation of surface-to-water $\mathrm{H}$-bonds, predominate over the cohesion ones (i.e. those stabilized by the water-water $\mathrm{H}$ bond network), thereby allowing water to wet large surface areas (Fig. 1A). When this occurs, surfaces of porous systems are indicated as having large water wettability, i.e. large attitude to interact with water molecules. If hydrophobic surfaces are present, water clusters keep the same number of $\mathrm{H}$-bonds as in the bulk (i.e. in the absence of the surface), and the water-surface interactions mostly occur then via van der Waals forces. ${ }^{[29]}$ As a result, the cohesion forces stabilizing the water-water $\mathrm{H}$-bond network are strengthened, and water molecules cannot be spread on large solid surface areas (Fig. 1B). If hydrophobicity predominates over hydrophilicity, water wettability decreases.

The measurement of the CA between a water droplet and a solid surface (Fig. 1) is the simplest routine experiment to retrieve information about solid surface nature (i.e. hydrophilic or hydrophobic) and its wettability. ${ }^{[26]}$ In fact, when CA is below $90^{\circ}$, hydrophilic surfaces with larger wettability must be accounted for (Fig. 1A). Conversely, CAs above $90^{\circ}$ indicate hydrophobic solid surfaces and lower wettability (Fig. 1B). ${ }^{[19,30]}$

The data reported in Table 1 show that CAs for the three biochar samples used in the present study are all above $90^{\circ}$, thereby suggesting that all the biochars have a hydrophobic nature. This conclusion accords with the results outlined in De Pasquale et al., ${ }^{[25]}$ where the same samples as studied here have been described as hydrophobic extended aromatic structures or graphite-like microcrystallites.

It is worth noting that although CAs reported in Table 1 are all above $90^{\circ}$, conifer biochar revealed a CA of $128^{\circ}$, which was significantly higher than those measured for poplar and grapevine marc biochars (both were $114^{\circ}$ ). This suggests that hydrophobicity of the former is larger than that of the latter two samples. In addition, we can also argue that both poplar and grapevine marc biochars reveal the same hydrophobic characteristics. According to the aforementioned discussion, we suggest that conifer biochar shows a lower wettability than that measured for both poplar and grapevine marc biochars, whose ability to interact with water molecules is, in turn, identical.

\section{FFC ${ }^{1} \mathrm{H}$ NMR experiments}

Figure 2 shows the NMRD profiles of the logarithmic average of the fastest relaxing water components in contact with the surfaces of the biochars obtained from conifer (Fig. 2A), poplar (Fig. 2B), and grapevine marc (Fig. 2C). As already reported in De Pasquale et al., ${ }^{[25]}$ the $R_{1}$ values varied in the order: $R_{1 \text { (conifer biochar) }}<R_{1 \text { (poplar biochar) }}<R_{1 \text { (grapevine marc biochar) }}$ in the whole $B_{R L X}$ range used in the present study.

It must be pointed out that the differences in NMRD shapes and $R_{1}$ values between the data reported in Fig. 2 and those discussed in De Pasquale et al. ${ }^{[25]}$ are due to the different $\tau$ period during which $B_{R L X}$ was applied (in the present study, this period was ten times longer than in De Pasquale et al. ${ }^{[25]}$ ) and to the diverse elaboration of the decay/recovery curves at each $B_{R L X}$ value (Materials and Methods).

The shorter aforementioned $\tau$ period applied in De Pasquale et al. ${ }^{[25]}$ prevented achievement of the longest relaxing water components, thereby revealing only the behavior of the relaxing time distribution of the water molecules closest to the biochar surface. Moreover, the use of the stretching function, accounting for the multi-exponential behaviors of the decay/recovery curves, allowed achievement of an average $T_{1}$ value (and, hence, an average $R_{1}$ value) at each $\mathrm{B}_{\mathrm{RLX}}$, which explained the overall NMR dispersion profile with only one model. ${ }^{[25]}$ In other words, both the short $\tau$ period during which $\mathrm{B}_{\mathrm{RLX}}$ was applied and the use of the stretching function for the decay/recovery curves elaboration, ${ }^{[25]}$ did not account for the details reported in the present study. Conversely, the longer $\tau$ period used here allowed the differentiation, via UPEN algorithm (Materials and Methods), between two different kinds of water molecules surrounding biochar solid particles. As already indicated in Materials and Methods, we only accounted for water molecules showing a NMR dispersion profile because of their direct interactions with biochars' surfaces.

De Pasquale et al. ${ }^{[25]}$ reported also that the NMRD profiles and the relaxograms of the water on the surface of the grapevine marc biochar sample could not be compared with those achieved for the water on poplar and conifer biochars because of the larger amount

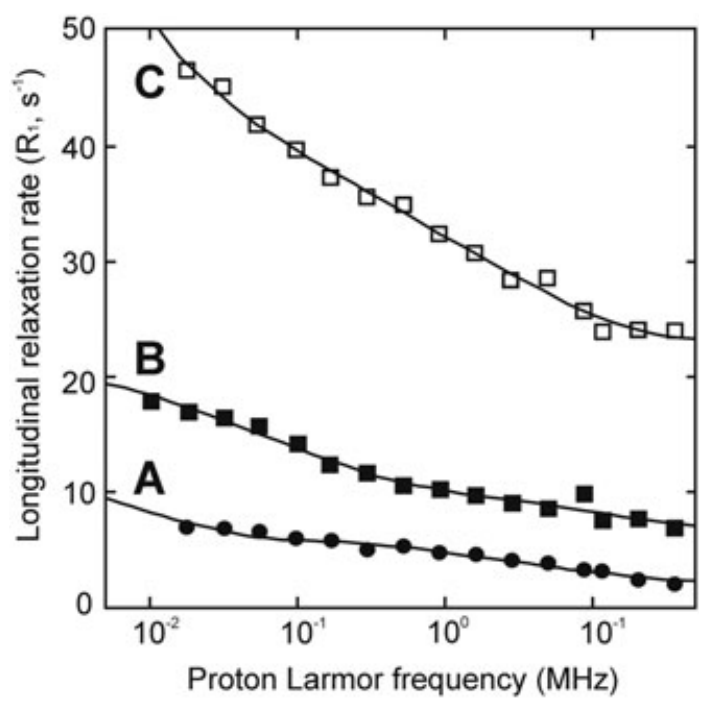

Figure 2. NMR dispersion profiles of the logarithmic average of the fastest relaxing water components in conifer biochar $(\mathbf{A})$, poplar biochar $(\mathbf{B})$, and grapevine marc biochar (C). 
of organic and inorganic paramagnetic species in the former than in the latter two samples. For this reason, any possible consideration on biochar pore structure and distribution through the application of the model-free analysis proposed by Halle and coworkers $^{[24]}$ was impossible for the grapevine marc biochar.

Table 1, reporting the NMR parameters indicated in the model from Korb and coworkers ${ }^{[23]}$ (Materials and Methods), confirms that the number of paramagnetic iron ions per gram of biochar $\left(\eta_{s}\right)$ is the largest in the grapevine marc biochar sample. However, the number of surficial iron ions $\left(\sigma_{s}\right)$ resulted in the order: $\sigma_{s \text { (conifer biochar) }}>\sigma_{s \text { (poplar biochar) }}>\sigma_{s \text { (grapevine marc biochar). }}$. Because of the fastest $R_{1 \text { (grapevine marc biochar) values in the whole }}$ $\mathrm{B}_{\mathrm{RLX}}$ range as compared with $R_{1 \text { (conifer biochar) }}$ and $R_{1 \text { (poplar biochar) }}$ (Fig. 2), we suggest that accessibility to paramagnetic sites is the largest in the biochar sample from grapevine marc. This accords with the lowest surface area $\left(S_{p}\right.$ in Table 1$)$ obtained by Brubauer-Emmet-Teller analyses. In fact, the lower the surface area, the larger is the size of the surface pores, ${ }^{[22]}$ thereby allowing easy penetration of water molecules and contact with the surficial paramagnetic centers.

As aforementioned, $\sigma_{s}$ values resulted in the order $\sigma_{s \text { (conifer biochar) }}$ $>\sigma_{s \text { (poplar biochar) }}$ (Table 1 ), while $R_{1}$ and $S_{p}$ values were in the order $R_{1 \text { (conifer biochar) }}<R_{1 \text { (poplar biochar) }}$ (Fig. 2A and 2B) and $S_{p \text { (conifer biochar) }}$ $<S_{p \text { (poplar biochar) }}$ (Table 1) for conifer and poplar biochars, respectively. These results confirm previous findings that poplar biochar is richer in small-sized pores, whereas large pore sizes appear to be characteristic for conifer biochar. ${ }^{[25]}$ In fact, if the mechanism of longitudinal relaxation was mainly affected by paramagnetism, we should have expected faster $R_{1}$ values for the conifer rather than for poplar biochar. Conversely, the $R_{1 \text { (conifer biochar) }}<R_{1 \text { (poplar biochar) }}$ observation ensures that the relaxation mechanism is dominated by the interactions between water molecules and the walls of the different sized biochar pores. In particular, the smaller pore sizes in the poplar biochar allow stronger solid-liquid interactions, thereby fastening proton longitudinal relaxation rates as compared with the water molecules in the larger pores of conifer biochar.

The continuous lines in Fig. 2 are the fitting curves obtained by the application of the model from Korb and coworkers ${ }^{[23]}$ as indicated in Eqn (2). This model contains the $\tau_{m}$ and $\tau_{s}$ values, which have been referred to as surface diffusion correlation time and surface residence time, respectively. ${ }^{[23]}$ In particular, the $\tau_{m}$ value measures the time spent by molecules to slip randomly among the binding sites on the biochar surface. ${ }^{[23,31]}$ The longer the $\tau_{m}$, the larger is the affinity of water molecules for the binding sites on the biochar surface. However, because of the weakness of the water-biochar surface interactions, ${ }^{[11]}$ water molecules may 'jump' from the surface towards a longer distance while they are replaced by other water systems. ${ }^{[23,31]}$ The time spent by water molecules for being fully decorrelated to the surface relaxing sites is the surface residence time. ${ }^{[23]}$ The shorter the $\tau_{s}$ value, the lower is the affinity of water for the biochar surface. Conversely, as water lays longer on biochar surface (longer $\tau_{s}$ values), larger surface hydrophilicity can be hypothesized. According to the aforementioned discussion, the $\tau_{s} / \tau_{m}$ ratio represents roughly the average number of diffusing steps of water molecules in proximity to the biochar binding sites (either organic or inorganic) during the time scale of the NMRD measurements. The larger this ratio is, the more numerous the $2 \mathrm{D}$ re-encounters are, and, therefore, the more correlated the water and biochar sites are. For this reason, $\tau_{s} / \tau_{m}$ ratio reveals the affinity of water molecules to biochar porous surface on the microscopic level, i.e. the dynamical surface affinity of the fluid for the pore surface.

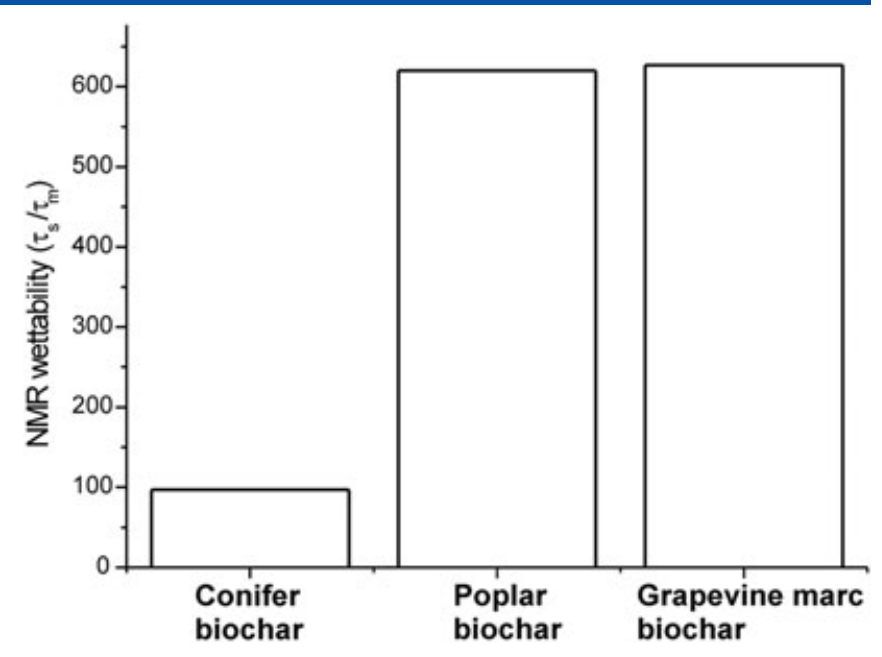

Figure 3. Nuclear magnetic resonance wettability obtained by fitting the NMR dispersion data in Fig. 2 with Eqn (1).

Figure 3 reports the $\tau_{s} / \tau_{m}$ ratios for the three biochars used in the present study. They resulted in the order: $\left(\tau_{s} / \tau_{m}\right)_{\text {conifer biochar }}<$ $\left(\tau_{s} / \tau_{m}\right)_{\text {poplar biochar }} \approx\left(\tau_{s} / \tau_{m}\right)_{\text {grapevine marc biochar. }}$ In other words, NMRrelated affinity of the biochar from conifer wastes was lower than that of the biochars from poplar and grapevine marc residues, respectively. The latter two samples showed, in turn, very similar NMR-related affinity values. These results suggest that the microscopic NMR-affinity can be related to the macroscopically observable wettability as measured by CA.

\section{Conclusions}

In the present study, we successfully measured the dynamical surface affinity of water in three different biochars (i.e. wettability) by FFC NMR relaxometry with the application of a new mathematical model developed by Korb and coworkers. ${ }^{[23]}$ Because of the similarity in wettability trends between the data by FFC NMR relaxometry and those by the traditional CA measurements, we suggest the use of the former technique as a valid and more reliable alternative to the latter one. In fact, the importance of NMR wettability relies on the possibility to monitor water dynamics at a nanometer length scale as compared with the macroscopic scale achieved by CA measurements. This allows prediction either on the possible transformation mechanisms occurring to biochar as it is applied to soils, or on nutrient availability when soils are enriched with biochar. ${ }^{[10]}$ Moreover, the agreement between the NMR relaxometry and CA measurements is the key result of our study, thereby suggesting a possible invariance in wettability mechanisms from a nano-scale up to a macro-scale dimension. Further studies are needed in order to confirm the results presented here and to evaluate the effect of biochar applications on wettability of biochar amended soils.

\section{Acknowledgements}

Dr. Alessandro Pozzi (AGT - Advanced Gasification Technolgy) and Dr. Massimo Valagussa (Fondazione Minoprio) are kindly acknowledged for having provided the biochar samples. Prof. Leonardo Palmisano and Dr. Vittorio Loddo from University of Palermo are acknowledged for having provided BET facilities. Dr. Dörte Diehl (University of Koblenz-Landau) is acknowledged for helping with CA measurements. This study was partially funded by 'Progetto FFR 2102/2013' financed by Università degli Studi di Palermo. 


\section{References}

[1] P. Conte, H.-P. Schmidt, G. Cimò, in Agricultural and Environmental Applications of Biochar: Advances and Barriers (Eds: M. Guo, Z. He, M. Uchimiya), SSSA Special Publication, vol. 63, SSSA, Madison, 2015; DOI: 10.2136/sssaspecpub63.2014.0050.

[2] M. Ogawa, Y. Okimori, F. Takahashi. Mitig. Adapt. Strat. Glob. Chang. 2006, 11, 429-444.

[3] D. A. Laird. Agron. J. 2008, 100, 178-181.

[4] J. A. Matthews. Energy Policy 2008, 36, 940-945.

[5] J. Lehmann, S. Joseph, Biochar for Environmental Management: Science and Technology, Earthscan, London, 2009.

[6] P. Conte, U. M. Hanke, V. Marsala, G. Cimò, G. Alonzo. J. Agric. Food Chem. 2014, 62, 4917-4923.

[7] G. Baiamonte, C. De Pasquale, V. Marsala, G. Cimò, G. Alonzo, G. Crescimanno, P. Conte. J. Soil Sediment 2015, 15, 816-824.

[8] P. Conte, N. Nestle, Magn. Reson. Chem. 2015; on-line-first DOI: $10.1002 /$ mrc.4204.

[9] J. Wang, X. Pan, Y. Liu, X. Zhang, Z. Xiong. Plant Soil 2012, 360, 287-298.

[10] C. I. Kammann, H.-P. Schmidt, N. Messerschmidt, S. Linsel, D. Steffens, M. Müller, H.-W. Koyro, P. Conte, S. Joseph. Sci. Rep. 2015, 5, 11080. doi:10.1038/srep11080.

[11] P. Conte, V. Marsala, C. De Pasquale, S. Bubici, M. Valagussa, A. Pozzi, G. Alonzo. GCB Bioenergy 2013, 5, 116-121.

[12] G. Cimò, J. Kucerik, A. E. Berns, G. E. Schaumann, G. Alonzo, P. Conte. J. Agric. Food Chem. 2014, 62, 1912-1918.

[13] D. Wang, W. Zhang, X. Hao, D. Zhou. Environ. Sci. Technol. 2013, 47, 821-828.

[14] S. Aston, A. Street-Perrott, S. Doerr, Geophysical Research Abstracts, EGU General Assembly 2014, vol. 16, 2014, EGU2014-12472-3.
[15] S. Yi, B. Witt, P. Chiu, M. Guo, P. Imhoff. J. Environ. Qual. 2015, 44, 963-971.

[16] E. de Blas, G. Almendros, J. Sanz. Geoderma 2013, 206, 75-84.

[17] D. Diehl. Colloids Surface Physicochem. Eng. Aspects 2013, 432, 8-18.

[18] M. Hajnos, A. Calka, G. Jozefaciuk. Geoderma 2013, 206, 63-69.

[19] Y. Yuan, T. R. Lee, in Surface Science Techniques. Springer Series in Surface Sciences, vol. 51 (Eds: G. Bracco, B. Holst), Springer-Verlag, Berlin, Heidelberg, 2013, pp. 3-33. doi:10.1007/978-3-642-34243-1.

[20] A. Simpson, M. Simpson, R. Soong. Environ. Sci. Tech. 2012, 46, $11488-11496$.

[21] J. Mao, X. Cao, N. Chen, in Advanced Biofuels and Bioproducts (Eds: J. W. Lee), Springer Science+Business Media, New York, 2013, pp. 47-55.

[22] P. Conte, G. Alonzo. eMagRes 2013, 2, 389-398.

[23] J.-P. Korb, M. Whaley-Hodges, R. G. Bryant. Phys. Rev. E 1997, 56, 1934-1945.

[24] B. Halle, H. Johanneson, K. Venu. J. Magn. Reson. 1998, 135, 1-13.

[25] C. De Pasquale, V. Marsala, A. E. Berns, M. Valagussa, A. Pozzi, G. Alonzo, P. Conte. J. Soil Sediment 2012, 12, 1211-1221.

[26] L. Wilhelmy. Annalen der Physik und Chemie 1863, 195, 177-217.

[27] N. B. Vargaftik, B. N. Volkov, L. D. Voljak. J. Phys. Chem. Ref. Data 1983, $12,817-820$

[28] A. Perera, R. Mazighi, B. Kežíc. J. Chem. Phys. 2012, 136, 174-516.

[29] V. V. Yaminsky, E. A. Vogler. Curr. Opin. Colloid Interface Sci. 2001, 6, 342-349.

[30] L. Románszki, M. Mohos, J. Telegdi, Z. Keresztes, L. Nyikos. Period. Polytech. Chem. Eng. 2014, 58, 53-59.

[31] P. J. McDonald, J.-P. Korb, J. Mitchell, L. Monteilhet. Phys. Rev. E 2005, 72, 011409. 